\title{
Trajectories of opioid consumption from day of surgery to 28 days postoperatively: a prospective cohort study in patients undergoing abdominal, joint, or spine surgery
}

\author{
Robert J McCarthy @ ( ) Ashley Meng Adams, Amanda C Sremac, Wendy Jo Kreider, \\ Pete L Pelletier, Asokumar Buvanendran
}

- Additional supplemental material is published online only. To view, please visit the journal online (http://dx.doi.org/ 10.1136/rapm-2021-102910).

Anesthesiology, Rush University, Chicago, Illinois, USA

Correspondence to

Dr Robert J McCarthy, Anesthesiology, Rush University, Chicago, Illinois, USA Robert_J_McCarthy@rush.edu

Received 28 May 2021 Accepted 10 September 2021 Published Online First 22 September 2021

Check for updates

(C) American Society of Regional Anesthesia \& Pain Medicine 2021. No commercial re-use. See rights and permissions. Published by BMJ.

To cite: McCarthy RJ, Adams AM, Sremac AC et al. Reg Anesth Pain Med 2021:46:1067-1075.

\section{ABSTRACT \\ Introduction Descriptions of opioid use trajectories and their association with postsurgical pain and opioid consumption are limited. We hypothesized that trajectories of opioid consumption in the first 28 days following surgery would be associated with unique patterns of pain and duration of opioid use.}

Methods A prospective longitudinal cohort of patients undergoing elective inpatient abdominal, joint, or spine surgery between June 2016 and June 2019 was studied. At hospital discharge and every 7 days for 28 days, patients were assessed for pain, analgesic use, pain interference, satisfaction, and side effects. Duration of opioid use was determined for 6 months. The primary analysis used latent class group modeling to identify trajectories of opioid use.

Results Decreasing, high, and persistent opioid trajectories were identified following joint and spine surgery and a decreasing and persistent trajectory following abdominal surgery. Reported pain was greater in the high and persistent trajectories compared with the decreasing use trajectories. Compared with the decreasing opioid trajectory, the median duration of opioid use was increased by 4.5 (95\% Cl 1 to 22 , $p<0.01$ ) weeks in persistent opioid use abdominal and by $6(95 \% \mathrm{Cl} 0$ to $6, p<0.01)$ weeks in the high or persistent use joint and spine groups. The odds $(95 \% \mathrm{Cl})$ of opioid use at 6 months in the high or persistent opioid use trajectory was 24.3 (2.9 to 203.4) for abdominal and 3.7 (1.9 to 7.0$)$ for joint or spine surgery compared with the decreasing use trajectory. Morphine milliequivalent per 24 hours of hospitalization was the primary independent predictor of opioid use trajectories. Conclusions We observed distinct opioid use trajectories following abdominal and joint or spine surgery that were associated with different patterns of pain and duration of opioid use postoperatively. Prediction of postoperative opioid use trajectory groups may be clinically important for identifying risk of prolonged opioid use.

\section{INTRODUCTION}

Following surgery, many patients experience moderate to severe pain which can interfere with return to daily activities of life. In the acute postoperative period ( $<7$ days), multimodal pain management including opioid, non-opioid, and regional anesthesia techniques is an important component of recovery following a surgical procedure. ${ }^{1}$ Unfortunately, inadequate treatment of acute postsurgical pain may negatively impact numerous aspects of patient health and contribute to the development of chronic postsurgical pain and persistent opioid use. $^{2-4}$

Prolonged postoperative opioid use following surgery is particularly concerning given the current opioid crisis in the USA. The Centers for Disease Control and Prevention cautions that long-term opioid use often begins with treatment of acute pain. ${ }^{5}$ In addition, unused prescription opioids also represent a significant problem as they may be diverted for non-medical use and contribute to the increasing burden of opioid-related injuries and death. Although policy efforts aimed at limiting opioid prescription quantities may help reduce the amount of unused opioids following surgery, they may limit effective pain management in some patients, increase patient suffering, and decrease satisfaction with pain management. ${ }^{67}$ A systematic review by Bicket et al found that more than two-thirds of patients reported unused prescription opioids following surgery. ${ }^{8}$ The aforementioned findings were consistent across studies of general, orthopedic, thoracic. and obstetric inpatient and outpatient surgeries. ${ }^{8}$

Unfortunately, a gap in knowledge exists regarding the patterns or trajectories of use of opioids in the early postoperative period ( $\leq 1 \mathrm{month}$ ) as well as the association of the opioid use patterns with reported pain and prolonged opioid use across various surgical procedures. Prior studies have evaluated procedural specific opioid use in the month to year following surgical procedure, ${ }^{7}$ but few have focused on identifying trajectories or patterns of use. In addition, studies frequently exclude high-risk patients, such as those taking preoperative opioid analgesics, and rarely evaluate opioid administration during hospitalization when assessing postoperative opioid use patterns. We hypothesized that unique trajectories or patterns of opioid use in the first month of surgery could be identified following surgical procedures of the abdomen, joints, and the spine and that these trajectories would display unique patterns of patient-reported pain and persistent ( $>6$ months) opioid use. Understanding these patterns and characteristics of patients in these groups may provide 
insight into patients at risk for long-term opioid consumption following surgery.

\section{METHODS}

This study was approved by the Institutional Review Board of Rush University (ORA\#16043002, IRB-02, approval date $5 / 20 / 2016)$. This manuscript adheres to the Strengthening the Reporting of Observational Studies in Epidemiology statement guidelines. All subjects provided informed written consent for study participation. The study design was a prospective, observational cohort conducted at Rush University Medical Center.

\section{Study population}

Eligible patients were English-speaking adults $\leq 85$ years of age undergoing a surgical procedure to a joint or bone (open reduction internal fixation, knee, shoulder, hip, or ankle) or the spine with an expected duration of admission of at least 24 hours that received opioid analgesics in the perioperative period and would receive a prescription for an opioid analgesic at the time of discharge. Excluded were patients undergoing non-included surgical procedures, current enrollment in another study, surgery for staging or treatment of cancer, patient being actively treated for a substance use disorder, and patient unwillingness to participate in study procedures including weekly phone assessment of pain and medication use.

Subjects were contacted by study personnel following surgery after the patient had been transferred to the hospital ward. Study personnel explained the study to the patient and obtained written informed consent for study participation. Just prior to hospital discharge, study personnel again contacted the patient and recorded the patient's response to questions regarding their average, minimum, and maximum pain during their hospital stay using the 11-point (0-10) numeric rating scale for pain (NRS) scale, with anchors of "no pain" at 0 and "worst pain ever" at 10. They also recorded the response to a question regarding how well the patient's pain was controlled and a question inquiring if the hospital staff did everything to help them with their pain on a 4-point Likert scale (Never, Sometimes, Usually, Always). Patients were then asked to reply using yes/no to the occurrence of side effects potentially attributable to analgesics during hospitalization. Discharge medications were reviewed with the patient as were their directions for use.

Subjects were contacted at 7 \pm 1 -day intervals for four consecutive periods ( 28 days) via phone calls by study personnel and their average, minimum, and maximum pain over the last 7 days were obtained using the NRS for pain scale. Subjects were also asked to rate interference with normal activities using a 5-point Likert scale (Never, Occasionally, Sometimes, Usually, Always). Analgesic medications taken over the last 7 days were recorded as were side effects potentially attributable to analgesics that occurred. Satisfaction with their pain medications was assessed on a 6-Point Likert scale (Very Dissatisfied, Dissatisfied, Slightly Dissatisfied, Slightly Satisfied, Satisfied, Very Satisfied). Patients were also questioned (yes/no) if they had unscheduled contact with their healthcare provider or gone to an immediate care facility in the last 7 days (online supplemental table 1).

\section{Covariates}

Patient sociodemographic characteristics were extracted from the medical records including: age, sex, race, ethnicity, and body mass index. Medical records were also evaluated for preoperative opioid analgesia use (within 30 days) by history and medication reconciliation. Surgical characteristics included type of surgery, American Society of Anesthesiologist physical status, and medications administered during surgery. Outcomes extracted from medical records included: pain assessments preoperatively and postoperatively until discharge, perioperative analgesics administered during the hospitalization, length of hospitalization, use of multimodal analgesia methods, and/or a regional anesthesia block. Multimodal analgesia was considered to include an opioid and any combination of one or more the following: a non-steroidal anti-inflammatory drug (excluding acetaminophen/opioid combination products), a gabapentinoid, an alpha-2 agonist, ketamine, or a local anesthetic. Opioid use after discharge was recorded as yes/no for weekly intervals up to or beyond 6 months from clinic notes, prescription refill information in the medical record and the Illinois Prescription Monitoring Program. ${ }^{9}$ The average pain score during hospitalization was calculated as the area under the NRS pain score by time curve using trapezoidal integration and dividing by the length of hospital stay. ${ }^{10} 11$ Opioid analgesics administered intraoperatively, during hospitalization and during the 4 weeks of follow-up, were converted to oral milligram morphine equivalents (MME) using the conversion table available from the National Center for Injury Prevention and Control. ${ }^{12}$ MMEs consumed during hospitalization were normalized to 24 hours of hospitalization to account for different lengths of stay. Because of the high heterogeneity in opioid use between the abdominal surgery and the joint and spine surgery subjects, we chose to separately perform latent class analysis on these two surgical groups.

\section{Primary and secondary outcomes}

The primary outcome of this study was opioid consumption from the day of surgery through 28 days postoperatively. Secondary outcomes included opioid use postdischarge up to or beyond 6 months, minimum, average, and maximum pain scores at discharge and the first 28 days following surgery, patient satisfaction with pain therapy and management, average reported pain during hospitalization, patient-reported side effects, and duration of hospital stay.

\section{Statistical analysis}

The primary analysis, a latent class trajectory modeling analysis was performed, using maximum likelihood estimation, and the LCTMtool package in R. ${ }^{13}$ The first step of the analysis was to examine the shape of the standardized residual plots in a model of no random effects. From the residual plot, a random quadric model was chosen. We chose $\mathrm{K}=5$ classes initially as this had been shown to be the number of pain trajectory classes during the first 7 days following similar surgeries. ${ }^{14}$ Evaluating the number of latent classes from 1 to 7 in the working model was then performed and the favored number of latent classes chosen based on the lowest Bayesian information criteria. The optimal structure of the model from fixed to unrestricted random effects was then determined. Using the final developed model individual patients were clustered into trajectory classes using the highest posterior probability of membership. The final selected model was assessed for adequacy by examining posterior probabilities of assignments, odds of correct assignment, and relative entropy. Mean trajectories and 95\% predictive intervals for each trajectory class were plotted to display the random variation within each class. Spaghetti plots of individuals in the latent classes were also plotted to examine overlap of subjects within the trajectory groups (figure 1). Discrimination of the trajectories was assessed using Elsensohn's envelope of residuals. ${ }^{15}$ Clinical 

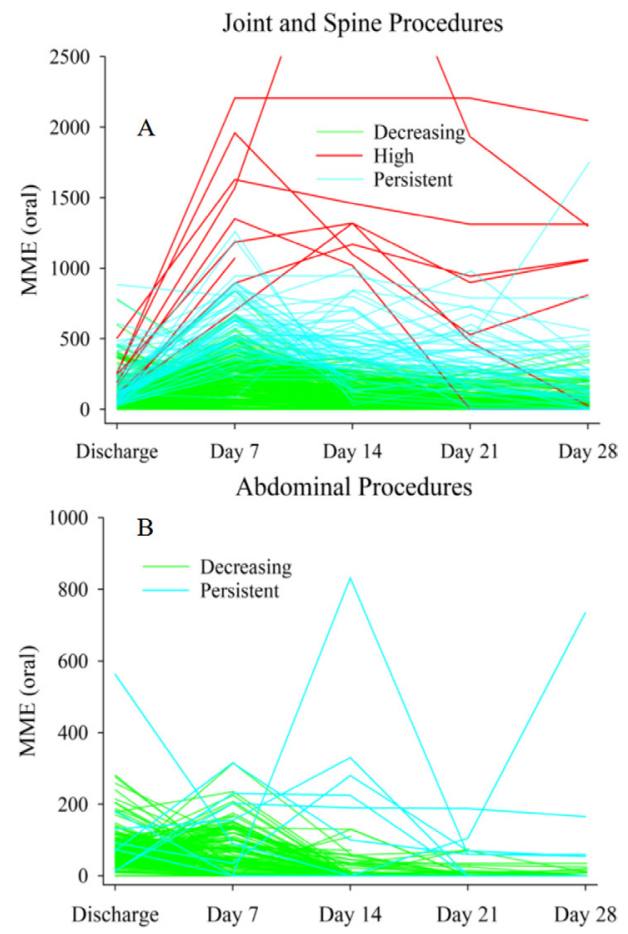

Figure 1 Spaghetti plots of individual patient MME use trajectories within the identified trajectory groups for joint and spine procedures and for abdominal procedures. Three trajectories were identified following joint and spine surgery: a decreasing opioid intake compared with the in-hospital utilization across the 28-day study, a high use group that had significantly increased opioid use during first 14 days following discharge compared with in-hospital utilization, and a persistent use group that had similar opioid use across the 28 days of the study. Two trajectories were identified following abdominal surgery: a decreasing use group and a persistent use group. MME, milligram morphine equivalents.
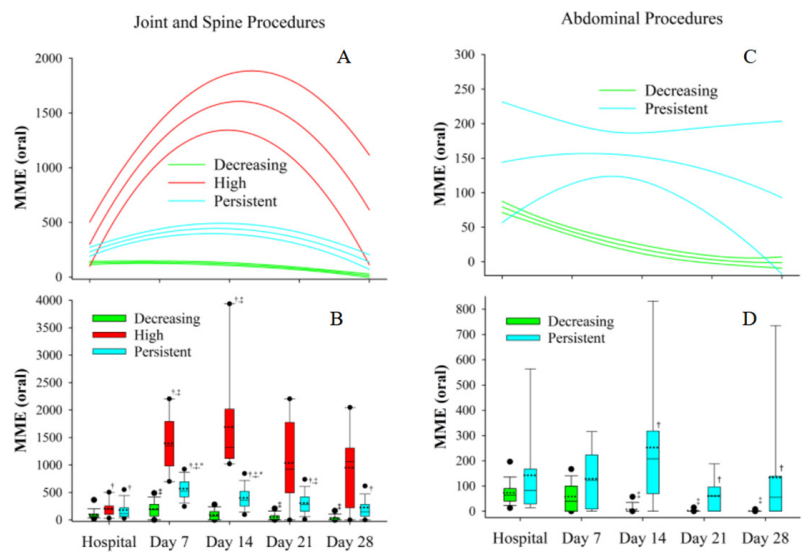

Figure 2 Opioid consumption trajectory estimates and 95\% prediction intervals for joint and spine procedures (Panel A) and abdominal surgery (Panel C). Panels B and D are box plots of opioid consumption reported at each of the five measurement points. Solid line in box is the median, dotted line is the mean, the upper and lower edges of the box represent the 25th and 75th percentiles, the whiskers represent the 10th and 90th percentiles, and the solid circle the 5th and 95th percentiles. * Different from high trajectory value at same time point. Differences at $p<0.05$ corrected for multiple comparison using the Bonferroni method. tDifferent from the decreasing trajectory value at same time point. $\ddagger$ Different from Hospital value within the same trajectory group. meaningfulness of the trajectory patterns along with inclusion of at least $1 \%$ of the sample in each of the identified classes was checked. Because latent class trajectory modeling using full information maximum likelihood estimation, patients with missing values were included in the models; however, patients that could not be contacted at any point following discharge were excluded from the analysis.

Continuous patient characteristic, surgical data, hospital pain data, and length of hospital stay were compared across latent classes using the Kruskal-Wallis $\mathrm{H}$ statistic or the MannWhitney U test. Posthoc analysis was performed using Dunn's test with Bonferroni correction. Differences in medians and CIs were determined using a 1000 sample bootstrap. Ordinal and nominal data were compared by creating cross-tabulation tables and a $\chi^{2}$ statistic. Differences in proportions and CIs were determined using the Clopper-Pearson method. In-hospital and 7-day average MME, minimum, average, and maximum patient reported pain scores were analyzed using generalized estimating equations with normal as the distribution and an identity link function fitted by maximum likelihood with patient ID as the subject variable and time of assessment (discharge, 7-day, 14-day, 21-day, and 28-day) as the within-subject variable and a firstorder autoregressive correlation matrix. Side effects reported were analyzed using generalized estimating equations with binomial as the distribution and a logit link function and an unstructured correlation matrix. Group, time, and group * time were assessed as factors in the generalized estimation equation analyses. Posthoc comparisons between pairs of data estimates were made using Bonferroni correction. Pain interference with daily activities and patient satisfaction with analgesic medications were analyzed using a $\chi^{2}$ statistic. Duration of opioid use was compared between trajectories using the log-rank test rightcensored at discontinuation of opioid use.

Age, sex, race, ethnicity, American Society of Anesthesiology physical status, preoperative opioid use, preoperative pain score, multimodal analgesia, regional anesthesia, tobacco use, alcohol use, average pain score during hospitalization, and the total opioid MME per 24 hours of hospitalization were entered into a logistic regression model for prediction of membership to an increased opioid use latent class trajectory group. A variable inflation factor $<5$ and a condition index $<20$ of the variables in the logistic model was required to limit multicollinearity. Model performance assessed using the Akaike information criteria and Nagelkerke's pseudo r-squared. The optimal cut-off value for the logistic regression models was selected as the point of maximization of the Kappa statistic. Model accuracy was determined by constructing a confusion matrix. A $\mathrm{p}<0.05$ was required to reject the null hypothesis.

Statistical analysis was performed using RStudio V.1.4.1717 (Integrated Development for R. RStudio, Boston, Massachusetts, USA; URL: http://wwwrstudio. com/) and R V.4.1.0, release date May 18, 2021 (The R Foundation for Statistical Computing, Vienna, Austria).

The sample size for this study was based on similar prospective studies examining pain trajectories and opioid consumption following surgeries. ${ }^{71617}$ No statistical power calculations were performed before this analysis.

\section{RESULTS}

Eighteen hundred and eighty subjects were approached and 705 provided written consent and were included in the study between June 2016 and June 2019. Excluded after providing consent were 10/705 (1.4\%) subjects who had surgical procedures not 


\section{Original research}

Table 1 Clinical characteristic, type of procedure, preoperative opioid use, use of multimodal and regional anesthesia/analgesia, in-hospital pain ratings, and length of stay by trajectory classes

\begin{tabular}{|c|c|c|c|c|c|c|c|}
\hline & \multicolumn{3}{|l|}{ Joint and spine } & \multirow[b]{2}{*}{$P$ value } & \multicolumn{2}{|l|}{ Abdominal surgery } & \multirow[b]{2}{*}{$P$ value } \\
\hline & $\begin{array}{l}\text { Decreasing } \\
(\mathrm{n}=328)\end{array}$ & High $(n=9)$ & Persistent $(n=68)$ & & Decreasing $(n=148)$ & Persistent $(n=8)$ & \\
\hline Sex, n (\%) & & & & 0.81 & & & 0.74 \\
\hline Male & $145(44)$ & $3(33)$ & $30(44)$ & & $25(17)$ & $1(13)$ & \\
\hline Female & $183(56)$ & $6(67)$ & $38(56)$ & & $123(83)$ & $7(87)$ & \\
\hline Median (IQR) age in years & $63(56-69)$ & $60(52-63)$ & $61(53-67)$ & 0.05 & $45(35-52)$ & $44(32-52)$ & 0.64 \\
\hline Median (IQR) BMI in $\mathrm{kg} / \mathrm{m}^{2}$ & $32.6(27.6-38.3)$ & $30.1(27.6-34.6)$ & $30.7(26.8-37.2)$ & 0.89 & $37.6(28.3-45.0)$ & $37.2(28.8-43.9)$ & 0.76 \\
\hline Race $n(\%)$ & & & & 0.14 & & & 0.85 \\
\hline White & $276(84)$ & $7(78)$ & $59(87)$ & & $85(57)$ & $4(50)$ & \\
\hline African American & $51(15)$ & $2(22)$ & $7(10)$ & & $61(41)$ & $4(50)$ & \\
\hline Asian & $1(1)$ & $0(0)$ & $2(3)$ & & $2(2)$ & $0(0)$ & \\
\hline Hispanic ethnicity n (\%) & $13(4)$ & $0(0)$ & $3(3)$ & 0.81 & $34(23)$ & $2(25)$ & 0.89 \\
\hline ASA physical class n (\%) & & & & 0.17 & & & 0.47 \\
\hline 1 & $18(5)$ & $0(0)$ & $2(3)$ & & $11(7)$ & $0(0)$ & \\
\hline 2 & $190(58)$ & $4(44)$ & $51(75)$ & & $81(55)$ & $6(75)$ & \\
\hline 3 & $120(37)$ & $5(56)$ & $15(22)$ & & $56(38)$ & $2(25$ & \\
\hline $\begin{array}{l}\text { Median (IQR) preoperative pain score } \\
\text { on a scale of } 0-10^{*}\end{array}$ & $2(0-5)$ & $6(2-8)$ & $3(0-6)$ & 0.03 & $0(0-0)$ & $0(0-3)$ & 0.62 \\
\hline Tobacco use $\mathrm{n}(\%)$ & & & & 0.42 & & & 0.87 \\
\hline Within last 2 years & $127(39)$ & $5(55)$ & $33(48)$ & & $47(38)$ & $3(37)$ & \\
\hline None or quit $>2$ years & $182(55)$ & $4(45)$ & $33(49)$ & & $90(61)$ & $5(63)$ & \\
\hline Missing & $19(6)$ & $0(0)$ & $2(3)$ & & $1(1)$ & $0(0)$ & \\
\hline Alcohol use $n(\%)$ & & & & 0.34 & & & 0.43 \\
\hline Frequent (>2 drinks per week) & $108(33)$ & $1(11)$ & $21(31)$ & & $32(22)$ & $1(12)$ & \\
\hline Infrequent ( $\leq 2$ drinks per week) & $99(30)$ & $6(67)$ & $25(37)$ & & $67(45)$ & $6(76)$ & \\
\hline None & $90(23)$ & $2(22)$ & $17(25)$ & & $47(32)$ & $1(12)$ & \\
\hline Missing & $31(10)$ & $0(0)$ & $5(7)$ & & $2(1)$ & 0 & \\
\hline $\begin{array}{l}\text { Preoperative opioid use (within } 30 \\
\text { days) } n(\%)\end{array}$ & $112(34)$ & $7(78)$ & $38(56)$ & $<0.01$ & $29(20)$ & $3(38)$ & 0.22 \\
\hline \multicolumn{8}{|l|}{ Type of surgery $n(\%)$} \\
\hline Abdominal & & & & & & & 0.23 \\
\hline Open & - & - & - & & $42(29)$ & $4(57)$ & \\
\hline Laparoscopic & - & - & - & & $92(62)$ & $3(43)$ & \\
\hline Robotic assisted & - & - & - & & $13(9)$ & $0(0)$ & \\
\hline Joint & & & & 0.44 & & & \\
\hline Hip & $83(33)$ & $1(25)$ & $11(20)$ & & - & - & \\
\hline Knee & $141(55)$ & $3(75)$ & $40(74)$ & & - & - & \\
\hline Shoulder & $8(3)$ & $0(0)$ & $0(0)$ & & - & - & \\
\hline Ankle & $5(2)$ & $0(0)$ & $1(2)$ & & - & - & \\
\hline Other & $17(7)$ & 00 & $3(4)$ & & - & - & \\
\hline Spine & & & & 0.45 & & & \\
\hline Cervical & $26(38)$ & $1(20)$ & $4(29)$ & & - & - & \\
\hline Thoracic/lumbar & $47(62)$ & $4(80)$ & $10(71)$ & & - & - & \\
\hline Multimodal analgesia $\mathrm{n}(\%)$ & $266(81)$ & $8(100)$ & $36(94)$ & 0.01 & $1014(69)$ & $6(75)$ & 0.73 \\
\hline Regional anesthesia/analgesia n (\%) & $101(31)$ & $1(11)$ & $36(53)$ & $<0.01$ & $62(42)$ & $3(38)$ & 0.81 \\
\hline $\begin{array}{l}\text { Median (IQR) average pain score } \\
\text { during hospitalization on a scale of } \\
0-10^{*}\end{array}$ & $3.3(1.8-4.7)$ & $6.0(2.9-7.0)$ & $3.7(1.7-5.3)$ & 0.02 & $4.0(2.9-5.2)$ & $4.8(4.3-6.8)$ & 0.03 \\
\hline Median (IQR) length of stay in hours & $48.9(28.7-73.1)$ & $91.9(54.6-159.4)$ & $33.1(26.4-57.6)$ & $<0.01$ & $43.7(28.8-56.6)$ & $69.5(50.8-136.8)$ & 0.09 \\
\hline
\end{tabular}

Data presented as $\mathrm{n}$ (\% of column) or median (first to third quartile). Ordinal and nominal data were analyzed by constructing cross-tabulation tables and using a $\chi^{2}$ test. Interval days were analyzed using the Kruskal-Wallis test (three groups) or the Mann-Whitney $U$ test (two groups).

${ }^{*}$ Assessed by care provider using the numeric rating score for pain, where $0=$ no pain and $10=$ worst pain imaginable.

ASA, American Society of Anesthesiology.

involving the abdomen, a bone or joint, or spine and 134/705 $(19.0 \%)$ subjects that were lost to follow-up at 7 -day postdischarge. All 561 patients included in the analysis received an opioid analgesic in the hospital, median (first to third quartile) MME per 24 hours 74 (48-113). The distribution of surgical procedures was: abdominal 156/561 (28\%), joint, and/or spine 
Table 2 Self-reported pain control, satisfaction and opioid related side effects at hospital discharge

\begin{tabular}{|c|c|c|c|c|c|c|c|c|}
\hline & \multicolumn{3}{|c|}{ Joint and spine surgery } & \multirow[b]{2}{*}{$P$ value } & \multicolumn{2}{|c|}{ Abdominal surgery } & \multirow[b]{2}{*}{$P$ value } \\
\hline & & $\begin{array}{l}\text { Decreasing } \\
(\mathrm{n}=328)\end{array}$ & High $(n=9)$ & $\begin{array}{l}\text { Persistent } \\
(\mathrm{n}=68)\end{array}$ & & $\begin{array}{l}\text { Decreasing } \\
(n=148)\end{array}$ & Persistent $(n=8)$ & \\
\hline \multirow{3}{*}{$\begin{array}{l}\text { Responses to the question of } \\
\text { "During your hospital stay } \\
\text { how often was your pain well } \\
\text { controlled?" n (\%) }\end{array}$} & Always & $189(58)$ & $3(34)$ & $35(51)$ & \multirow[t]{3}{*}{0.45} & $81(55)$ & $2(25)$ & \multirow[t]{3}{*}{0.01} \\
\hline & Sometimes & $44(13)$ & $3(33)$ & $10(15)$ & & $15(10)$ & $4(50)$ & \\
\hline & Never & $5(2)$ & $0(0)$ & $0(0)$ & & $1(1)$ & $0(0)$ & \\
\hline \multirow{2}{*}{$\begin{array}{l}\text { Responses to the question of } \\
\text { "During your hospital stay, how } \\
\text { often did the hospital staff do } \\
\text { everything they could to help } \\
\text { you with your pain? n (\%) }\end{array}$} & Sometimes & $4(1)$ & $0(0)$ & $2(3)$ & \multirow{2}{*}{0.95} & $2(2)$ & $0(0)$ & \multirow{2}{*}{0.67} \\
\hline & Never & $1(0)$ & $0(0)$ & $0(0)$ & & $0(0)$ & $0(0)$ & \\
\hline \multirow{3}{*}{$\begin{array}{l}\text { Responses to the question of } \\
\text { how satisfied are you with } \\
\text { your pain medications during } \\
\text { hospitalization? } \mathrm{n}(\%)\end{array}$} & Very satisfied & $176(54)$ & $5(56)$ & $21(34)$ & \multirow[t]{3}{*}{0.02} & $85(57)$ & $2(25)$ & \multirow[t]{3}{*}{$<0.01$} \\
\hline & Satisfied & $106(33)$ & $2(22)$ & $29(46)$ & & $48(32)$ & $4(50)$ & \\
\hline & Slightly satisfied & $26(8)$ & $0(0)$ & $7(11)$ & & $11(7)$ & $0(0)$ & \\
\hline \multicolumn{9}{|c|}{$\begin{array}{l}\text { Opioid related side effects during hospitalization } \mathrm{n} \\
(\%)\end{array}$} \\
\hline Dizziness & & $111(35)$ & $1(11)$ & $19(28)$ & 0.189 & $60(41)$ & $2(25)$ & 0.479 \\
\hline Nausea & & $84(26)$ & $1(11)$ & $12(18)$ & 0.247 & $62(42)$ & $3(38)$ & 0.806 \\
\hline Vomiting & & $44(13)$ & $0(0)$ & $5(8)$ & 0.201 & $36(24)$ & $2(25)$ & 0.965 \\
\hline Constipation & & $104(32)$ & $3(33)$ & $16(24)$ & 0.403 & $17(12)$ & $2(25)$ & 0.255 \\
\hline Difficulty urinating & & $46(14)$ & $1(11)$ & $4(6)$ & 0.182 & $52(35)$ & $2(25)$ & 0.715 \\
\hline Abdominal discomfort & & $28(9)$ & $2(22)$ & $4(6)$ & 0.246 & $69(47)$ & $3(38)$ & 0.728 \\
\hline Drowsiness & & $208(63)$ & $9(100)$ & $43(63)$ & 0.077 & $112(72)$ & $7(88)$ & 0.681 \\
\hline Sleeplessness & & $141(43)$ & $3(33)$ & $21(31)$ & 0.163 & $43(29)$ & $3(38)$ & 0.694 \\
\hline Pruritus & & $73(22)$ & $2(22)$ & $23(34)$ & 0.127 & $30(20)$ & $1(12)$ & 0.592 \\
\hline
\end{tabular}

Data presented as $n$ (\% of column). Ordinal and nominal data were analyzed by constructing cross-tabulation tables and using a $\chi^{2}$ test.

surgery $405 / 561$ (72\%). The list of included surgical procedures is shown in online supplemental table 2.

Eighteen hundred and eighty-three of 2205 (93\%) potential MME values were used in the latent modeling of opioid use following joint and spine surgery. Three trajectories of opioid consumption (decreasing, high, and persistent) from hospitalization through the 4-week postdischarge were identified following joint and spine surgery (figure 2A). Opioid consumption per 24 hours of hospitalization and for the first 28 days postoperatively is shown in figure $2 \mathrm{~B}$. Decreasing trajectory subjects (328/405, 81\%) MME use was lower during hospitalization and at days 7 and 14 compared with subjects in high (9/405, 2\%) and persistent $(68 / 405,17 \%)$ groups. Median (first to third quartile) total opioid use in MME following discharge among the joint and spine surgery trajectories was 302 (120 to 560) MME for the decreasing trajectory, 2525 (1625 to 7187) MME for the high trajectory, and 1260 (1054 to 1640) for the persistent trajectory groups. The median $(99 \% \mathrm{CI})$ difference in postdischarge MME use was 2223 (886 to 8405) MME for the high versus the decreasing trajectory, 958 (855 to 1178) MME for the persistent versus the decreasing trajectory, and $-1265(-7407$ to 147$)$ for the high versus the persistent trajectory group.

Seven hundred and fifty-eight of 780 (97\%) potential MME values were used in the latent modeling of opioid following abdominal procedures. Two trajectories of opioid consumption (decreasing and persistent) were identified following abdominal surgery (figure 2C). Opioid use was greater on days 14, 21, and
28 for persistent $(8 / 156,5 \%)$ compared with the decreasing $(148 / 156,5 \%)$ trajectory group (figure 2D). Median (first to third quartile) total opioid use in MME following discharge among the abdominal surgery trajectories was 45 (0 to 130) MME for the decreasing trajectory, and 508 (444 to 810) MME for the persistent trajectory groups. The median (99\% CI) difference in postdischarge MME use was 463 (215 to 796) MME for the persistent vs the decreasing trajectory groups.

Clinical characteristic, surgical types, preoperative opioid use, use of multimodal and regional anesthesia/analgesia, in-hospital pain ratings, and length of stay by latent class trajectory groups are shown in table 1 . In the joint and spine surgery group, the high trajectory group had greater preoperative opioid use, multimodal analgesia use, average pain during hospitalization, and longer length of stay compared with decreasing trajectory patients. Persistent trajectory patients had greater preoperative pain scores, preoperative opioid use, and multimodal and regional anesthesia use compared with the decreasing trajectory group. There were no differences between the high and persistent trajectory groups. In the abdominal surgery subjects, persistent trajectory subjects had higher pain scores during hospitalization compared with the decreasing trajectory group.

Self-reported pain control, satisfaction, and opioid-related side effects by patient report at hospital discharge are shown in table 2. Abdominal surgery subjects in the persistent trajectory reported less well-controlled pain during hospitalization compared with the decreasing trajectory group, whereas 


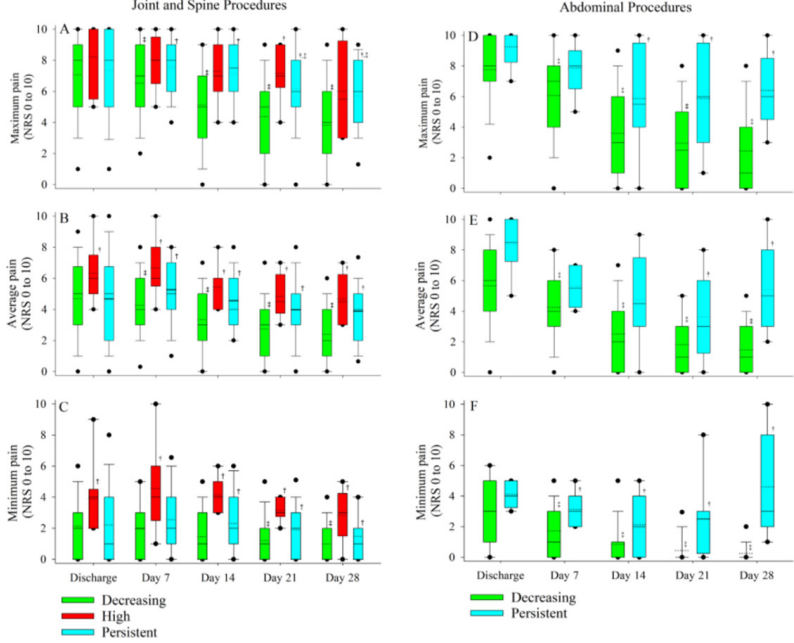

Figure 3 Box plots of reported pain reported at each of the five measurement points. Solid line in box is the median, dashed line is the mean, the upper and lower edges of the box represent the 25th and 75th percentiles, the whiskers represent the 10th and 90th percentiles, and the solid circle is the 5th and 95th percentile. Panels A and D: Maximum pain reported at discharge from hospitalization and then for the last 7 days at days 7, 14, 21, and 28. Panels B and E: Average pain reported at discharge from hospitalization and then for the last 7 days at days 7, 14, 21, and 28. Panels $C$ and F: Minimum pain reported at discharge from hospitalization and then for the last 7 days at days 7 , 14,21 , and 28. Differences at $p<0.05$ corrected for multiple comparison using the Bonferroni method. †Different from the decreasing trajectory group value at same time point. $¥ D$ ifferent from discharge value within the same trajectory group.

persistent trajectory subjects in the joint and spine group reported less satisfaction with pain medications compared with decreasing trajectory subjects.

Patient-reported maximum, average, and minimum pain during hospitalization reported at discharge and at days 7, 14, 21 and 28 postdischarge are shown in figure 3 . In joint and spine surgery, subjects in the high and persistent trajectories reported greater average pain at each 7-day interval compared with decreasing trajectory subjects. Maximum pain was greater in the persistent trajectory group compared with the decreasing trajectory and minimum pain was greater in the high and persistent groups from day 14 onward. The abdominal surgery persistent trajectory group reported greater minimal pain at all 7-day intervals postdischarge, maximal pain from day 14 onward, and greater average pain at days 21 and 28 .

Pain interference with daily activities and satisfaction with pain medication following discharge are shown in table 3. Pain interference with daily activities was greater in the persistent trajectory group at days 7,14, and 21 and at 21 days in high trajectory group compared with the decreasing trajectory group following joint and spine procedures. Following abdominal procedures, pain interference was greater at days 21 and 28 in the persistent trajectory group. Satisfaction with pain medications was reduced in both the high and persistent trajectories in the joint and spine group and the persistent trajectory of the abdominal group compared with the corresponding decreasing trajectory group.

Self-reported side effects and unscheduled visits to the healthcare system following discharge are shown in online supplemental tables 2 and 3. Persistent trajectory subjects in the joint and spine surgery group reported a greater incidence of nausea at days 14 and 21, greater constipation at day 21, and a greater incidence of contact with their medical provider at day 7 compared with decreasing trajectory subjects. There were no differences in reported side effects or contact with the healthcare system between the trajectory groups in the abdominal surgery groups.

The median (first to third quartile) length of opioid use in the joint and spine surgery procedures groups were 4 (2 to 10 ) weeks, 10 (10 to 17) weeks, and 10 (10 to 18 ) weeks for the decreasing, high, and persistent trajectories, respectively. The median difference $(99 \% \mathrm{CI})$ in opioid use between the high and decreasing trajectory groups was 6 ( 0 to 20 ) weeks, $p=0.04$, and 6 ( 0 to 6 ) weeks, $\mathrm{p}<0.01$, between the persistent and decreasing trajectories. In the abdominal surgery subjects, the median length of opioid use in the decreasing trajectory group was 2 (1 to 3 ) weeks and 6.5 (3 to 22.5 ) weeks in the persistent trajectory, median difference 4.5 ( 1 to 22$)$ weeks, $(p<0.01)$.

Continued opioid use at 6 months following surgery was identified in $46 / 405(11 \%)$ of subjects in the joint and spine and $4 / 156(3 \%)$ of the abdominal surgery groups. The OR $(95 \% \mathrm{CI})$ of continued opioid use at 6 months in the high or persistent trajectory group compared with decreasing trajectory group was 3.65 (1.90 to $7.00, \mathrm{p}<0.01)$ following joint and spine surgery. Following abdominal surgery, the OR for continued opioid use at 6 months was $24.2(2.97$ to $203.38, \mathrm{p}<0.01)$ for persistent compared with the decreasing trajectory group. Seventeen subjects had potential confounding reasons for opioid use at 6 months: eight had subsequent surgery, four were receiving treatment for cancer, and five had new painful medical conditions (stroke, ruptured quadricep muscle, and three had new herniated disc disease). Removing these subjects increased the OR of continued opioid use for the high or persistent trajectory groups compared with the decreasing group following joint and spine surgery to 4.31 (95\% CI 2.00 to $9.04, \mathrm{p}<0.01)$. The OR for persistent opioid use in the abdominal surgery group compared with the decreasing group was unchanged.

Table 4 shows the results of the multivariable regression analyses. Binominal regression analysis identified preoperative opioid use, multimodal analgesia, and hospital MME consumption per 24 hours as independent predicators for identifying high or persistent trajectory subjects compared with decreasing trajectory subjects following joint and spine surgery. The model area under the receiver operating characteristic curve (ROC) was $77 \%$ (95\% CI $71 \%$ to $82 \%)$. At a predicted probably cutoff of 0.254 , the Kappa statistic was 0.29 (95\% CI 0.21 to $0.38)$, sensitivity $(\mathrm{SE})$, specificity $(\mathrm{Sp})$, positive predictive value (PPV), and negative predictive value (NPV) of the model were 56\%, 80\%, 39\%, and 88\%, respectively. In abdominal surgery patients, hospital MME consumption per 24 hours and length of stay were identified as an independent predictor of persistent trajectory group membership. The model area under the ROC was $80 \%$ (95\% CI $62 \%$ to $98 \%)$. At a predicted probably cutoff of 0.254 , the Kappa statistic was 0.31 (95\% CI 0. to 0.58), sensitivity (SE), specificity (Sp), PPV, and NPV of the model were $37 \%, 98 \%, 60 \%$, and 97\%, respectively.

\section{DISCUSSION}

The important findings of this study are that latent class trajectory modeling of opioid consumption from the day of surgery through 4 weeks postoperatively identified unique trajectory groups of patients that demonstrated clinically different pain profiles and clinically important differences in postdischarge opioid consumption over the first 28 days following 
Table 3 Responses to questions regarding pain interference and satisfaction with analgesic medications at days 7, 14, 21, and 28

\begin{tabular}{|c|c|c|c|c|c|c|c|c|}
\hline & & \multicolumn{3}{|c|}{ Joint and spine surgery } & \multirow[b]{2}{*}{$P$ value } & \multicolumn{2}{|c|}{ Abdominal surgery } & \multirow[b]{2}{*}{$P$ value } \\
\hline & & Decreasing & High & Persistent & & Decreasing & Persistent & \\
\hline \multicolumn{9}{|c|}{ Responses to the question "How does Pain Interfere with Normal Activities" $n$ (\%) } \\
\hline \multirow[t]{3}{*}{ Day 7} & Always or usually & $112(35)$ & $5(55)$ & $42(62)$ & \multirow[t]{3}{*}{$<0.01$} & $26(18)$ & $3(37)$ & \multirow[t]{3}{*}{0.185} \\
\hline & Sometimes & $69(21)$ & $3(33)$ & $13(19)$ & & $40(28)$ & $2(25)$ & \\
\hline & Occasionally or never & $140(44)$ & $1(12)$ & $13(19)$ & & $76(56)$ & $3(38)$ & \\
\hline \multirow[t]{3}{*}{ Day 14} & Always or usually & $59(20)$ & $3(43)$ & $35(56)$ & \multirow[t]{3}{*}{$<0.01$} & $8(6)$ & $2(25)$ & \multirow[t]{3}{*}{0.11} \\
\hline & Sometimes & $61(20)$ & $2(29)$ & $13(21)$ & & $18(13)$ & $2(25)$ & \\
\hline & Occasionally or never & $176(60)$ & $2(28)$ & $14(23)$ & & $115(81)$ & $4(50)$ & \\
\hline \multirow[t]{3}{*}{ Day 21} & Always or usually & $32(12)$ & $2(33)$ & $24(42)$ & \multirow[t]{3}{*}{$<0.01$} & $6(4)$ & $2(40)$ & \multirow[t]{3}{*}{0.1} \\
\hline & Sometimes & $55(21)$ & $2(33)$ & $11(19)$ & & $10(8)$ & $0(0)$ & \\
\hline & Occasionally or never & $175(67)$ & $2(34)$ & $22(39)$ & & $104(86)$ & $3(60)$ & \\
\hline \multirow[t]{3}{*}{ Day 28} & Always or usually & $31(13)$ & $3(50)$ & $15(29)$ & \multirow[t]{3}{*}{$<0.01$} & $3(3)$ & $2(40)$ & \multirow[t]{3}{*}{$<0.01$} \\
\hline & Sometimes & $37(15)$ & $1(16)$ & $10(19)$ & & $6(5)$ & $2(40)$ & \\
\hline & Occasionally or never & $173(72)$ & $2(34)$ & $27(52)$ & & $99(92)$ & $1(20)$ & \\
\hline \multicolumn{9}{|c|}{ Responses to the question "How Satisfied are you with your Pain Medications" $n$ (\%) } \\
\hline \multirow[t]{3}{*}{ Day 7} & Very satisfied or satisfied & $279(87)$ & $6(66)$ & $47(71)$ & $<0.01$ & $119(89)$ & $5(63)$ & 0.08 \\
\hline & Slightly satisfied or slightly dissatisfied & $29(9)$ & $3(33)$ & $19(28)$ & & $11(28$ & $2(25)$ & \\
\hline & Dissatisfied or very dissatisfied & $13(4)$ & $0(0)$ & $1(1)$ & & $5(3)$ & $1(12)$ & \\
\hline \multirow[t]{3}{*}{ Day 14} & Very satisfied or satisfied & $267(92)$ & $4(58)$ & $44(72)$ & $<0.01$ & $103(94)$ & $6(75)$ & $<0.01$ \\
\hline & Slightly satisfied or slightly dissatisfied & $14(5)$ & $3(42)$ & $15(23)$ & & $6(5)$ & $1(13)$ & \\
\hline & Dissatisfied or very dissatisfied & $9(3)$ & $0(0)$ & $3(5)$ & & $1(1)$ & $1(12)$ & \\
\hline \multirow[t]{3}{*}{ Day 21} & Very satisfied or satisfied & $227(92)$ & $4(67)$ & $43(77)$ & $<0.01$ & $92(97)$ & $5(63)$ & $<0.01$ \\
\hline & Slightly satisfied or slightly dissatisfied & $14(6)$ & $2(33)$ & $10(18)$ & & $2(2)$ & $1(12)$ & \\
\hline & Dissatisfied or very dissatisfied & $5(2)$ & $0(0)$ & $5(5)$ & & $1(1)$ & $2(25)$ & \\
\hline \multirow[t]{3}{*}{ Day 28} & Very satisfied or satisfied & $199(92)$ & $4(66)$ & $39(75)$ & $<0.01$ & $74(96)$ & $2(34)$ & $<0.01$ \\
\hline & Slightly satisfied or slightly dissatisfied & $13(6)$ & $2(34)$ & $12(23)$ & & $2(2)$ & $2(34)$ & \\
\hline & Dissatisfied or very dissatisfied & $2(2)$ & $0(0)$ & $1(2)$ & & $2(2)$ & $2(34)$ & \\
\hline
\end{tabular}

Data presented as $n(\%)$ of columns. Ordinal data were analyzed by constructing cross-tabulation tables and using a $\chi^{2}$ test.

surgery. High and persistent use opioid consumption trajectories following joint and spine surgery as well as persistent use following abdominal surgery were associated with longer postdischarge opioid consumption as well as higher odds of opioid use beyond 6 months. Subjects in the high or persistent opioid use trajectories reported greater pain, greater pain interference with daily activities, and less satisfaction with their analgesic regimen.

Given that the OR $(95 \% \mathrm{CI})$ of preoperative opioid use for being in a high or persistent opioid use trajectory in the joint and spine procedures was 2.0 (1.13 to 3.55$)$ and 28.1 (0.87 to 381 ) in the abdominal surgery group, opioid tolerance may partially explain the decreased analgesia and satisfaction in these groups despite the high use of multimodal analgesia methods; however, other unknown or unmeasured genetic or behavioral factors in these patients may be responsible for these differences.

Prior studies have examined opioid consumption following the surgical procedures included in this study. Larach et al evaluated patient factors associated with opioid consumption in the month after surgery in patients undergoing hysterectomy, thoracic surgery, and total knee and hip arthroplasty in opioid naïve patients and found that younger age, non-white race, lack of a college degree, higher anxiety, greater sleep disturbance, heavy alcohol use, current tobacco use, and a larger initial opioid prescription were risk factors for increase opioid consumption. ${ }^{7}$ Procedures were evaluated individually, reconciliation of opioid use was made only at 1 month, and in-hospital use was nonevaluated. Unlike the current study, the investigators did not attempt to evaluate patterns or trajectories of opioid use among or unique to the surgical groups.

In the current study, we found in-hospital opioid use to be the most significant predictor of opioid use trajectory assignment following both joint and spine as well as abdominal surgery procedures. Prior studies have shown that inpatient opioid use predicts postdischarge use in the first month following surgery. Lovecchio et al prospectively examined opioid use and pain scores for up to 6 weeks after surgery. ${ }^{18}$ They divided the group into low and high opioid consumers at the median number of pills consumed. They found that factors that have been repeatedly associated with the development of chronic opioid use after orthopedic surgery, such as psychiatric history and previous opioid use, were not associated with greater opioid consumption in the acute postoperative period. Bartels et al sought to identify characteristics of patients who reported using half or more of the opioid prescribed to them at discharge in a cohort undergoing cesarean delivery and one undergoing thoracic surgery. ${ }^{19}$ In both cohorts, they found that the use of opioids while hospitalized was greater in the group that reported using greater use of opioids after discharge. Emerson et al, following women for 2-5 weeks after cesarean delivery, found that the amount of opioid pain medication used during hospitalization was associated with the amount of opioid pain medication consumed after hospital discharge. ${ }^{20}$ Similar to our findings, a systematic review of 33 studies found that preoperative use of opioids and pain before surgery are predictors of greater opioid use in the postoperative period. $^{21}$ 
Table 4 Logistic regression analysis for identification of membership to a high or persistent use opioid trajectory

\begin{tabular}{|c|c|c|c|c|c|c|}
\hline & Joint an & surge & & Abdom & surgery & \\
\hline & $\boldsymbol{\beta}$ & OR & $95 \% \mathrm{Cl}$ & $\boldsymbol{\beta}$ & $\mathrm{OR}$ & $95 \% \mathrm{Cl}$ \\
\hline Sex & & & & & & \\
\hline Male & & 1 & & & 1 & \\
\hline Female & 0.029 & 1.03 & 0.57 to 1.86 & -1.404 & 0.27 & 0.00 to 17.4 \\
\hline Age (years) & -0.006 & 0.66 & 0.97 to 1.02 & -1.591 & 0.98 & 0.85 to 1.11 \\
\hline Race & & & & & & \\
\hline White & & 1 & & & 1 & \\
\hline African American Race & -0.426 & 0.65 & 0.27 to 1.51 & 0.438 & 1.55 & 0.07 to 38.5 \\
\hline Hispanic ethnicity & -0.31 & 0.73 & 0.15 to 2.65 & -0.831 & 0.43 & 0.02 to 8.0 \\
\hline Tobacco use & & & & & & \\
\hline Within 2 years & 0.439 & 1.55 & 0.58 to 3.88 & 0.765 & 0 & - \\
\hline Quit $>2$ years & 0.488 & 1.63 & 0.88 to 3.02 & -0.167 & 2.14 & 0.13 to 41.1 \\
\hline Alcohol use & & & & & & \\
\hline Frequent (>2 drinks per week) & -0.132 & 0.88 & 0.42 to 1.82 & -1.402 & 0.25 & 0.00 to 133.5 \\
\hline Infrequent ( $\leq 2$ drinks per week) & 0.387 & 1.47 & 0.76 to 2.89 & 7.364 & 157.8 & 4.8 to 2505.8 \\
\hline ASA physical status & & & & & & \\
\hline I or II & & 1 & & & 1 & \\
\hline III & -0.494 & 0.61 & 0.31 to 1.14 & -0.762 & 0.47 & 0.13 to 9.7 \\
\hline Preoperative opioid use & 0.696 & 2 & 1.14 to 3.55 & 3.33 & 28.1 & 0.87 to 381.8 \\
\hline Preoperative pain score $(0-10)$ scale & 0.061 & 1.06 & 0.97 to 1.17 & -0.006 & 0.96 & 0.40 to 1.75 \\
\hline Multimodal analgesia & 1.25 & 3.49 & 1.24 to 12.64 & 0.315 & 1.37 & 0.07 to 46.7 \\
\hline Regional anesthesia/analgesia & 0.582 & 1.79 & 0.95 to 3.38 & 0.659 & 1.93 & 0.09 to 45.5 \\
\hline Average pain score during hospitalization (on a 0-10 scale) & 0.172 & 1.18 & 0.99 to 1.41 & 0.246 & 1.28 & 0.51 to 3.24 \\
\hline MME use per 24 hours of hospitalization & 0.004 & 1 & 1.00 to 1.01 & 0.002 & 1.02 & 1.00 to 1.05 \\
\hline Length of hospitalization (h) & 0.002 & 1 & 0.99 to 1.01 & 0.119 & 1.13 & 1.04 to 1.30 \\
\hline
\end{tabular}

ASA, American Society of Anesthesiology.

In a study using similar methods to the current study, Vasilopoulos et al evaluated pain trajectories in a mixed surgical cohort across days 1-7 following surgery. ${ }^{14}$ Five distinct trajectories of pain were identified: low, moderate-to-low, moderate-to-high, and high pain over time. A fifth trajectory identified dramatically decreasing pain over time. No association was found with preoperative or intraoperative opioid use after correction for multiple comparisons; however, postoperative opioid use was significantly associated with the pain trajectory groups. Together, the current study and that of Vasilopoulos support the association of pain and opioid use trajectories in the postoperative period.

The results of our study should only be interpreted in the context of its limitations. We included subjects undergoing a diverse array of surgeries in an attempt to find generalizable patterns of opioid consumption; however, the heterogeneity in subject characteristics and opioid use between abdominal and joint and spine subjects suggest that this approach may be constrained by patterns of use that are surgical procedurespecific. We limited our sample to subjects that were admitted to the hospital for at least 24 hours postoperatively which may represent a selection bias in subject selection. Our abdominal surgery sample as well as the number of subjects in the high opioid use was small, limiting inclusion of variable in the multivariable model. We did not include psychological factors such as depression, anxiety, and pain catastrophizing that have been previously been shown to influence postoperative opioid use; however, we did include patients with known risk of increased opioid consumption such as preoperative opioid use and preoperative pain that have frequently been excluded in prior studies. We did not limit or adjust opioid prescriptions provided to the patients per protocol, which may influence patterns of postoperative use.

\section{CONCLUSION}

Our findings suggest that distinct trajectories of opioid use which can be characterized as either high or low use exist following surgery involving the abdomen, joints, and the spine. These trajectories are associated with different patterns of reported pain, interference in activities of daily life, and satisfaction with pain medication. Opioid use during hospitalization is the primary independent predictor of high use trajectories with preoperative opioid use also playing an important role. Further studies are needed to better understand factors associate with opioid use trajectories following surgery.

Twitter Pete L Pelletier @PetePelletierMD

Acknowledgements The authors would like to thank Mahendra Shah M.D. for his help in data acquisition for this study.

Contributors RJM was involved in the oversight of the study, acquisition and analysis of the data, drafting the article, critical revision of the article, and final approval of the version to be published. AMA was involved in the acquisition of the data, drafting the article, critical revision of the article, and final approval of the version to be published. ACS was involved in the acquisition of the data, drafting the article, critical revision of the article, and final approval of the version to be published. WJK was involved in the acquisition of the data, drafting the article, critical revision of the article, and final approval of the version to be published. PLP was involved in the conception or design of the study, drafting the article, critical revision of the article, and final approval of the version to be published. $A B$ was involved in the conception or design of the study, drafting the article, critical revision of the article, and final approval of the version to be published.

Funding This study was supported by Department of Anesthesiology internal funding. 
Competing interests None declared.

Patient consent for publication Not applicable.

Ethics approval Institutional Review Board of Rush University (ORA\#16043002, IRB-02, approval date 5/20/2016.

Provenance and peer review Not commissioned; externally peer reviewed.

Data availability statement Data are available on reasonable request. Deidentified participant may be available from RJM (ORIC id:0000-0002-

0966-5311), the corresponding author, on request and execution of a data use agreement.

ORCID iD

Robert J McCarthy http://orcid.org/0000-0002-0966-5311

\section{REFERENCES}

1 Wardhan R, Chelly J. Recent advances in acute pain management: understanding the mechanisms of acute pain, the prescription of opioids, and the role of multimodal pain therapy. F1000Res 2017;6:2065.

2 Buvanendran A, Della Valle CJ, Kroin JS, et al. Acute postoperative pain is an independent predictor of chronic postsurgical pain following total knee arthroplasty at 6 months: a prospective cohort study. Reg Anesth Pain Med 2019;44:e100036.

3 Alam A, Gomes T, Zheng H, et al. Long-term analgesic use after low-risk surgery: a retrospective cohort study. Arch Intern Med 2012;172:425-30.

4 Sinatra R. Causes and consequences of inadequate management of acute pain. Pain Med 2010;11:1859-71.

5 Centers for Disease Control and Prevention. Guideline for prescribing opioids for chronic pain. Available: http://www.cdc.gov/mmwr/volumes/65/rr/rr6501e1.htm [Accessed 17 May 2021].

6 Howard R, Vu J, Lee J, et al. A pathway for developing postoperative opioid prescribing best practices. Ann Surg 2020;271:86-93.

7 Larach DB, Sahara MJ, As-Sanie S, et al. Patient factors associated with opioid consumption in the month following major surgery. Ann Surg 2021;273:507-15.

8 Bicket MC, Long JJ, Pronovost PJ, et al. Prescription opioid analgesics commonly unused after surgery: a systematic review. JAMA Surg 2017;152:1066-71.
9 Illinois prescription monitoring program. Available: https://www.ilpmp.org/CDC/login. php [Accessed 07 Jul 2021].

10 Matthews JN, Altman DG, Campbell MJ, et al. Analysis of serial measurements in medical research. BMJ 1990:300:230-5.

11 Bell ML, King MT, Fairclough DL. Bias in area under the curve for longitudinal clinical trials with missing patient reported outcome data: summary measures versus summary statistics. Sage Open 2014;4.

12 National Center for Injury Prevention and Control. Cdc compilation of benzodiazepines, muscle relaxants, stimulants, zolpidem, and opioid analgesics with oral morphine milligram equivalent conversion factors, 2016 version. Atlanta, GA: Centers for Disease Control and Prevention, 2016. https://wwwcdcgov/drugoverdose/ medial

13 Lennon $\mathrm{H}$, Kelly S, Sperrin M, et al. Framework to construct and interpret latent class trajectory modelling. BMJ Open 2018;8:e020683.

14 Vasilopoulos T, Wardhan R, Rashidi P, et al. Patient and procedural determinants of postoperative pain trajectories. Anesthesiology 2021;134:421-34.

15 Elsensohn M-H, Klich A, Ecochard R, et al. A graphical method to assess distribution assumption in group-based trajectory models. Stat Methods Med Res 2016:25:968-82.

16 Hah JM, Cramer E, Hilmoe H, et al. Factors associated with acute pain estimation, postoperative pain resolution, opioid cessation, and recovery: secondary analysis of a randomized clinical trial. JAMA Netw Open 2019;2:e190168.

17 Kurteva S, Tamblyn R, Khosrow-Khavar F, et al. Postoperative duration of opioid use and acute healthcare services use in cancer patients hospitalized for thoracic surgery. J Surg Oncol 2021;124:431-40.

18 Lovecchio F, Premkumar A, Stepan JG, et al. Opioid consumption patterns after lumbar Microdiscectomy or decompression. Spine 2019:44:1599-605.

19 Bartels K, Mayes LM, Dingmann C, et al. Opioid use and storage patterns by patients after hospital discharge following surgery. PLoS One 2016;11:e0147972.

20 Emerson JB, Danilack VA, Kulkarni A, et al. Outpatient opioid use after cesarean delivery. R I Med J 2020;103:68-74.

21 Lawal OD, Gold J, Murthy A, et al. Rate and risk factors associated with prolonged opioid use after surgery: a systematic review and meta-analysis. JAMA Netw Open 2020;3:e207367 